\title{
From evolution to revolution: understanding mutability in large and disruptive human groups
}

\author{
Roger M. Whitaker ${ }^{a}$, Diane Felmlee ${ }^{b}$, Dinesh C. Verma ${ }^{c}$, Alun Preece ${ }^{a}$, Grace-Rose Williams ${ }^{d}$ \\ ${ }^{a}$ School of Computer Science \& Informatics, Cardiff University, Cardiff, UK CF24 3AA \\ ${ }^{b}$ Dept. of Sociology \& Criminology, Pennsylvania State University, PA, USA 16802 \\ ${ }^{c}$ IBM T.J. Watson Research Center, 1101 Kitchawan Road, Yorktown Heights, NY, USA 10598 \\ ${ }^{d}$ Defence Science \& Technology Laboratory, Porton Down, Salisbury, Wiltshire, U.K, SP4 0JQ
}

\begin{abstract}
Over the last 70 years there has been a major shift in the threats to global peace. While the 1950's and 1960's were characterised by the cold war and the arms race, many security threats are now characterised by group behaviours that are disruptive, subversive or extreme. In many cases such groups are loosely and chaotically organised, but their ideals are sociologically and psychologically embedded in group members to the extent that the group represents a major threat. As a result, insights into how human groups form, emerge and change are critical, but surprisingly limited insights into the mutability of human groups exist. In this paper we argue that important clues to understand the mutability of groups come from examining the evolutionary origins of human behaviour. In particular, groups have been instrumental in human evolution, used as a basis to derive survival advantage, leaving all humans with a basic disposition to navigate the world through social networking and managing their presence in a group. From this analysis we present five critical features of social groups that govern mutability, relating to social norms, individual standing, status rivalry, ingroup bias and cooperation. We argue that understanding how these five dimensions interact and evolve can provide new insights into group mutation and evolution. Importantly, these features lend themselves to digital modeling. Therefore computational simulation can support generative exploration of groups and the discovery of latent factors, relevant to both internal group and external group modelling. Finally we consider the role of online social media in relation to understanding the mutability of groups. This can play an active role in supporting collective behaviour, and analysis of social media in the context of the five dimensions of group mutability provides a fresh basis to interpret the forces affecting groups.
\end{abstract}

Keywords: group modelling, mutability, sociology, psychology

\section{INTRODUCTION}

It is well-recognised that the common threats to world peace have dramatically changed over the last 70 years. The beginning of this period was dominated by the cold war and its associated arms race. Clearly defined by identifiable actors, formal administrations, territorial boundaries and observable behaviour, the cold war allowed analysts and strategists to successfully model rational behaviour and understand the dynamics through game theory. ${ }^{1}$ While not directly diminishing the threat, the resultant insights offered an important contribution for policy makers and stakeholders to rationalise the conflict and assess the outcomes of strategy.

Moving to the present day, the cold war threats have been replaced by instabilities that are much more challenging to explicitly model and understand. ${ }^{2}$ Obfuscated by the human dynamics involving a large number of dispersed and often hidden actors, the disruptive behaviour of subversive and extreme groups now threaten regional stability in numerous locations across the globe, being characterized as asymmetric warfare. Unlike the cold war where advancement in nuclear technology supported a singular threat, these groups are often regressive, reverting to primitive forms of warfare and ideals, employing forms of violence spanning from hate to terror, combined with use modern technology such as social media to reach beyond their physical group. This behaviour is often accompanied by informal and chaotic internal group organisation, accompanied by reinforcement of the group's ideology through sanctions that could be extreme.

Send correspondence to R.M. Whitaker, Email: whitakerrm@cardiff.ac.uk, Tel: +44 (0) 2920876999 
The seemingly chaotic nature of such groups is confounded by their ability to quickly establish, adapt their character, fragment and combine. Broadly speaking, we refer to the associated dynamics as mutability, representing the ways in which these groups emerge and change. The problem of understanding important instabilities in world security today therefore involves understanding the mutability of groups. However, defining a single unifying framework for group behaviour is unlikely to be feasible. Instead it is more realistic to identify important concepts in sociology and psychology that translate into the computational domain and which allow us to investigate how mutability occurs in large groups. This is a complex challenge that spans many disciplines, and would benefit from an overarching strategy.

In this paper we argue that important factors in understanding the mutability of groups arise from examining the primeval origins of human behaviour. Specifically human evolution has been predicated on sustaining and exploiting large groups, and thousands of generations of natural selection have left humans with a basic disposition to navigate the world through social networking and managing their presence in a group, through which they can benefit from a coalition. From considering the origins of human social groups, we present five critical features through which changes in groups can be framed, modeled and assessed. These concern social norms, individual standing, status rivalry, ingroup bias and cooperation, around which an individual's social decision making occurs and collective behaviours emerge.

While these features are implicit in previous narratives (e.g. ${ }^{3}$ ), in this paper we examine and relate these features. As they can be computationally represented, we argue that harnessing them through digital modeling can support causal explanations (rather than correlative ones) for group evolution. Furthermore, such modelling supports the identification of possible latent factors that may affect the evolution of group behaviour. Finally we consider the role of online social media in relation to understanding the mutability of groups. In particular this media can play an active role in supporting group mutability, and analysis of social media in the context of the proposed mutable dimensions provides a fresh basis to interpret the features of groups.

\subsection{Examples of current approaches to modelling groups}

Modelling a group is challenging: groups are composed of many individuals that are complex in their own right, with the brain providing an advanced "cognitive architecture" that moderates how individuals perceive and respond to the world. In particular the brain enables complex and higher level functions such as consciousness (e.g., implicit information fusion ${ }^{4}$ ), self-awareness (e.g., identity ${ }^{5}$ ) and feelings (e.g., emotional intelligence ${ }^{6}$ ) that augment and influence personal reasoning. These powerful functions allow human beings to navigate the world by extrapolating sophisticated interactions with each other. This leads to distinctive collective behaviours, many of which are captured through sociological and psychological theory, providing a basis for modelling groups with greater realism to possible scenarios. However this pursuit is very much in its infancy.

While individual behaviours contribute to the existence and characteristics of a group, a group itself represents more than the sum of its individual members. In everyday life there are may examples where individuals would be unlikely to an act in a particular manner without the presence, both in terms of influence and solidarity, of a group: protests and picket lines are well-used examples. An immediate question arising is how one should attempt to model this, so that the dynamics of social forces can be better understood. This represents a continuum between modelling the interactions of individual actors independent of groups, as opposed to recognising a group as an entity in its own right with characteristics capable of influence or action on others.

Based on extending isolated individual behaviours, modelling psychological interactions between actors has been an important approach. One of the most important contributions in this regard has been Social Impact Theory, proposed by Latané. ${ }^{7}$ This has provided a useful quantitative framework that creates agency between individuals based on their psychological influence. This is based on four dimensions: the degree to which an individual is influenced by and influences others; the ability to persuade people with opposing beliefs to change their views; the ability to support others with similar beliefs and their position in the social structure.

Social Impact Theory has been useful in examining social interactions in specific contexts such as opinion formation, beliefs and culture. ${ }^{8,9}$ This general approach follows modelling in computational theoretical physics, where effectively people replace particles, and macro-level phenomenon are observed from micro-level interaction that is forced by physical constraints (e.g., time, space). Through an associated set of governing equations, 
Social Impact Theory has achieved convenient quantification of potential psychological behaviour. However further flexibility in modelling behaviour, constraints and features, including those of groups is highly desirable. This can be achieved though extending the cognition that agents possess, and the social attributes included in the model. ${ }^{10-12}$

Alexrod's agent-based modelling approaches (e.g., ${ }^{13,14}$ ) demonstrate this to good effect. A particularly interesting example ${ }^{15}$ includes group identity as a dormant trait, whose presence is determined to be an important latent feature in the emergence of ethnocentrism. Alexrod's work also highlights the importance of evolutionary modelling, specifically that as well as biological inheritance of characteristics, cultural effects can take place through social learning. Observing such evolution in simplified models has identified strong underlying rules that are sufficient to influence group behaviour. Modelling with simplicity appears to have been an important factor: additional parameters that could be sought in the pursuit of a closeness to reality haven't detracted from observing fundamental effects.

At the other end of the individual-group modelling spectrum is the biologically inspired "Blau space". ${ }^{16}$ This model involves a vector of socio-demographic variables for each participant and characteristics of groups. The approach functions based on homophily - that is those with similar socio-demographic characteristics are more likely to be associated by involvement in similar groups. Blau spaces project this information and find equilibrium between potentially competing groups, using a system of differential equations to derive a competition matrix between finite resources (individual participation). This avoids extensive data collection about the social network relations, and allows generative analysis of changes to demographics. This approach also circumvents the eventbased modelling approach, where features arise as the consequence of actions, as seen in Social Impact Theory and the evolutionary insights of Axelrod.

System dynamics has also been adopted as a useful means to understand the non-linear relationships behind the forces influencing group behaviour. ${ }^{17}$ This approach involves defining a bounded problem scenario and eliciting the "stocks and flows" which are the abstract representations of virtual (or physical) commodities and their local connection and influence. Originating from industrial dynamics this approach allows for the effects of feedback to be observed for scenarios where linear models are insufficient. System dynamics can be applied at a high level where individuals are not explicitly recognised, and instead the relationships between groups take precedence. Hybrid approaches to modelling have also emerged, ${ }^{18,19}$ which seek to span the modelling spectrum. These seek to combine multiple cognitive models capturing individual decision making with feedback and influence from a wider context, through systems dynamics. With such layered complexity embedded within the model, accuracy and effectiveness becomes a function of correctly parameterising and weighting a wide range of variables.

Social Impact Theory, the work of Axelrod, Blau spaces and system dynamics are exemplars demonstrating how social and behavioural insights can be gleaned through computational models. The generative nature of these methodologies is attractive ${ }^{20}$ because it enables social forecasting for specific scenarios. This can support and augment experts and qualitative approaches such as the Delphi method. ${ }^{21}$ However, concerning group behaviour, current progress in forecasting is at a formative stage. In scientific areas such as weather and climate, where great improvements in forecasting of natural systems are evident, progress has been made from combined advances in modelling, observational data and computational resources. Turning to social forecasting, advances in each of these areas are now equally possible, with developments in modelling being fundamental.

\subsection{The emerging challenge - characterising groups}

A particular imperative concerns establishing conventions that allow groups, as well as individuals, to be explicitly understood in terms of fundamental attributes, features and interactions within such models. Understanding which theories are most relevant, the balance between competing theories and the applicability of subjective or qualitative frameworks remains open. Arguably these are weaknesses that carry over to the current computational modelling literature, where the dominant frame of reference is the individual actor rather than a group. This is divergent from theory in sociology and psychology that explicitly recognises the role of groups and their identity as fundamental. For example, the act of showing commitment to a group, regardless of its rationality, can become an overriding goal for those that are entirely dependent on their role within that group. ${ }^{22}$ Group identities come 
into play in this context, and are known to dominate over an individuals identity particularly in situations of intergroup conflict.

Better computational understanding of groups as entities in their own right is needed for assessment of dynamic situations. For example, explicitly modelling groups as a unit of scale allows simulation to change resolution, and focuses on the agency held by a group, which comes into play when individuals are disposed to interacting based on their group's identity, such as in disruptive and extreme scenarios. ${ }^{23,24}$ This is required so that we can make group-level comparisons (both inter-group and intra-group), to better understand group mutability and assess high-level generative scenarios.

However this raises the question as to the most appropriate and useful dimensions to characterise groups. Furthermore, establishing dimensions that capture the essence of human groups is a challenge in itself. Logically though, the origin, purpose and function of groups from an evolutionary perspective offers a rationale as to why groups are useful to humans. The role that groups have played in evolution is significant and groups have a profound influence on the rationality of human behaviour. ${ }^{22}$ Therefore to establish useful dimensions to assess mutability, we revert to examining the origins of human groups.

\section{THE ORIGIN OF HUMAN GROUPS}

The modern human, or homo-sapien primate, emerged around 250,000 years ago, intertwined with the arrival of language $\mathrm{e}^{25}$ which allowed the species to sustain larger groups compared to their ancestors. Consistent with this, humans approach and frame their interaction with others in today's world as a consequence of thousands of years of evolution, that has conditioned the traits on which behaviour is predicated. In particular humans are distinguished among primates by their extreme prosocial disposition, ${ }^{26}$ seeking to gain security and advantage in their world based on interactions with others. This is embedded in human culture, ${ }^{27}$ evolving from ancestors who were Pleistocene hunter-gatherers. ${ }^{28}$ Here the benefits of coalitions were felt from defence and hunting in an environment fraught with the risk of predator attack. ${ }^{3}$

When resources were scarce and threats significant, the ability to successfully work in groups provided a means to increase survival prospects. ${ }^{29}$ Without establishing bonds for sound relationships, individuals were more vulnerable to free-riders and exploitation, which is easier to monitor when groups are small. ${ }^{30}$ Consequently bonding with others, and donating time to show commitment, ${ }^{31}$ have been a fundamental element of creating and sustaining relationships. ${ }^{32}$ Clusters of such tightly connected relationships in our ancestral groups provided a community defence where allies had an increased obligation to faithfully service all their interconnected relationships.

The formation and sustenance of relationships is often seen today based on bonding through commonality of some description. Known as homophily, ${ }^{33}$ this results in groups being defined around cultural issues, as well as geographical factors and extended kinship. This means that groups can potentially define and function as entities that represent ideologies and practices where individuals within them act and respond under their influence. ${ }^{24}$ Interestingly however, complex barriers exist that prevent convergence to a single group that has a unified cultural view: ${ }^{14}$ for example polarisation was found to increase when there are limited attributes associated with the culture available to a population. Understanding how such polarisation manifests itself, particularly in extreme forms, therefore remains important for the future.

\subsection{The Social Brain}

The capacity for a group to emerge and change is driven by the cognitive behaviour of its members in response to the context that they face both internally and externally, from potential collaborators and adversaries, as well as the group itself. ${ }^{34}$ This cognitive capacity has been shaped by the need for humans to survive in a group setting, resulting in distinctive social cognition, commonly called the human social brain. ${ }^{35}$

Prospering in large groups is not straight forward: as a group enlarges, the defence system provided by a tightly knit social network weakens because maintaining relationships with many others is a major cognitive challenge $^{36}$ and social knowledge from direct observation isn't always possible. This gives opportunity to freeriders, who can exploit others and move on before their reputation catches up with them through communication in a social network. Consequently individuals that are more advanced in judging others based on limited feedback, 
or those who have cognitive capabilities to sustain large social networks, are generally less likely to be exploited, thereby protecting their resources and enhancing chances of reproductive success. In other words, social cognition is likely to have acted as a selection pressure.

The complexity of the social cognitive challenge of assessing others is reflected in the large relative size of the human brain as compared to other species. Humans live in the largest social groups of all primates, typically around 150 in size. ${ }^{37} \mathrm{~A}$ fundamental correlation has been identified that shows a relationship between the social complexity of a species and the associated neocortex ratio. ${ }^{38}$ This represents the frontal lobes of the brain that are responsible for social activity, as a proportion of the total brain size. When taking the typical group size that a species maintains as a proxy for social complexity, this has led to the Social Brain Hypothesis, ${ }^{39}$ which argues that human intelligence evolved as a means of surviving and reproducing in large and complex groups.

After 250,000 years of homo-sapien evolution, where natural selection has been influenced by social intelligence, the brain now provides an instinctive compulsion for humans to understand the world from a social perspective. $^{40}$ We think about ourselves in relation to those around us with remarkable insight. For example, we can preempt thinking of people we know, ${ }^{41}$ ascribe intentions ${ }^{42}$ and make wide ranging social judgements. ${ }^{43}$ We can connect with the pleasure or pain felt by others and anticipate their position and views. ${ }^{44}$ Furthermore, social cognition is the default state for the brain, ${ }^{45}$ leading us to naturally ruminate about others. Therefore social reasoning plays an important role in how groups emerge and mutate. Combined with a wide range of factors such as individual differences, culture, social learning and context, there is significant complexity to consider. Therefore frameworks through which groups can be considered are highly valuable.

\section{DIMENSIONS FOR CONSIDERING GROUP MUTABILITY}

The evolutionary background to social groups shows how humans have used groups as a means to promote survival prospects. Although in the contemporary literature there is no singular and universal model that describes how "modern humans" interact in groups, arguably some of the most profound and original sociological understanding in this area was framed by George Homans. ${ }^{46}$ Holmans' thesis resonates with the human evolutionary origins, where he identifies factors such as control, authority, reciprocity and ritual as fundamental elements through which the outcomes of social interaction can be examined. This was continued by Homans' further work ${ }^{47}$ where $^{2}$ he used reinforcement and exchange as the basis to examine cooperation. This work remains highly relevant today, and is influential in our framing of the factors through which groups mutate. We hypothesise that (at least) five dimensions interact and support group evolution, namely social norms, individual standing, status rivalry, ingroup bias and cooperation. We present these in the following Sections (Sections 3.2-3.5), and propose these dimensions as ways in which the essence of groups can be characterised, assessed and compared.

\subsection{Social norms}

Social norms are critical to the functioning of groups and the dynamics between groups. These are the often unspoken and unwritten rules that represent acceptable behaviour to a collection of people, encompassing their expectations and values, as viewed from their own perspective. Norms indicate what a group expects as "normal" behaviour and contribute to reducing uncertainty on the rules and social actions that a society follows. ${ }^{48}$

Social norms represent a group-level belief system, acknowledged as a powerful sociological and psychological phenomenon. ${ }^{34}$ These are readily observed in societies and groups, being fundamental to the culture that a group sustains. ${ }^{49}$ However explaining why people comply with social norms and even promote or enforce them, particularly when this involves resource implications to the individual, ${ }^{50}$ has remained a central puzzle in social theory, ${ }^{34}$ social psychology ${ }^{51}$ and theoretical biology. ${ }^{52}$ Although this picture is still being built and has many facets, research has addressed aspects such as conditions for their stability and enforcement within a group. ${ }^{13}$

Characterising the social norms of a group is fundamentally important because they represent the potential impact of a group's behaviour on itself and others, including the consequences of non-compliance. Of all the different types of behaviour that a social norm may encompass, arguably the most important are the behavioural expectations towards their own members (i.e., ingroup) and others beyond (i.e., outgroup), as further considered in Section 3.4. These define the extent of a tolerant, and functional society, and relations between groups. 
Social norms naturally emerge, but may be enforced on a population as an instrument of control, such as in an authoritarian or insurgent regime.

Based on common belief and values, a social norm may itself define a group and attract membership, or equally norms may culturally emerge from within a pre-existing group fuelled by ongoing interactions or similarity of traits. It is normally the case that individuals will belong to a wide range of different groups, ranging from demographics, interests and other characteristics. Social identity theory ${ }^{53}$ proposes that an individual's sense of identity is driven by the groups to which they belong. Originating in $^{24}$ Tajfel et al propose that the pursuit of social identity through groups occurs as a consequence of seeking self-enhancement, particularly concerning belief supporting superiority of one's own group (ingroup bias), and uncertainty reduction, by using group affiliation as a proxy to represent one's beliefs and communicate this to others.

Although social norms exist in many forms, often being both culturally important and benign (e.g., lifestyle related such as fashion or music), extreme and disruptive group behaviours can also emerge, representing a significant violation of decency and humanity. These may sustain forms of aggression, from discrimination through to violence and conflict, which in turn can range from hate through to terrorism. In these extreme cases, social norms represent a threat to security and freedom, which is a feature of current day warfare.

Modelling of social norms in this context remains at a formative stage. Ethnographic contributions have enabled insight into the process by which individuals become psychologically induced into groups characterised by extreme norms, ${ }^{54,55}$ while established political insights have examined the discourse that accompanies the emergence of such groups and their relations. ${ }^{24}$

\subsection{Individual standing}

Within a group, despite often seeing commonality among members, it is rarely the case that individuals are respected equally by their peers or external group members. Individual standing captures how an individual is thought about from the perspective of a third party, with respect to trusting another to be faithful to particular social norms (Section 3.1). Stratification may contribute to this, representing a grouping with which an individual identifies. Action to promote standing and in response to standing fuel ingroup dynamics. Standing is assessed by the extent of one's reputation, which is the perceived evidence supporting ones belief about another person's intentions. The group(s) to which an individual belongs may also influence this, and the human predisposition is to more highly value affiliation with ones own group (Section 3.4).

Reputation assessment is an instinctive human pursuit that triggers neurological effects. ${ }^{56}$ It informs trust in others and more widely it supports the concept of intentionality, ${ }^{42}$ which is the inference of motives and beliefs that people hold in respect of each other. Intentionality functions as the belief system allowing humans to socially hypothesize, a distinguishing feature of human cognition. Within this, comparing reputation plays a significant role in how people choose to understand each other and themselves (Section 3.3), allowing humans to engage in analysis and make wide ranging social judgements. ${ }^{43}$ This is critical to internal group rivalry (Section 3.3), where individuals compete to obtain power within a group.

In sociology reputation has received little systematic attention, ${ }^{57}$ with wide ranging alternative definitions. However definitions often tend to capture the idea of making an assessment of future behaviour based on past history. This has high relevance in economics where investment decision making can be influenced. ${ }^{58}$ In this context reputation effectively holds the role of a currency, acting as a memory and asset that allows the resources of others to be accessed in future, making reputation management strategically important to the individual. ${ }^{59}$

In a group context, commentators note ${ }^{60}$ that reputation plays an important role in alliance formation to sustain cooperation in groups populated beyond genetic relatives. Here reputation acts as a signalling system to a wider population who may not have a history of direct observation from previous interactions. This is particularly important when donating resources to help a third party, and thus arises in cooperative scenarios (Section 3.5). Informed decision making is important, both to ensure one's own resources are conserved and that one's own reputation in the eyes of others is not diminished by inappropriate behaviour towards others. This will be shaped in a group by the social norms (Section 3.1) which may extend to cooperative expectations. 


\subsection{Status rivalry}

Status rivalry, reflecting competition for social power and dominance, can occur between individuals in a group or between groups (Section 3.4). When rivalry escalates within a group, this can be accompanied with conflict and potential division into new groups. The dynamic that supports this is fuelled by the social capital that adversaries can lever from within the group. Social capital broadly relates to the resources that are accumulated through cooperative relationships held with others, reflecting an individual positioning within a social network. Knowing the nature of relationships with other people determines ones position with regard to social capital within a social network, informing the commitment that can be meaningfully devoted towards others, as well as informing our expectations of others and determining their position.

Social capital is predicated on investment in social relations with expected returns. ${ }^{61}$ Its values lies in facilitation of information, exertion is influence of agents and in signaling the social credentials or standing (Section 3.2). Authors such as Burt ${ }^{62}$ and Bourdieu ${ }^{63}$ have explored the sociological and economic implications of social capital and the reader is referred to associated works for more in depth analysis. However, from a group perspective, social capital is broadly characterised as bridging and bonding, based on potential link strength with another party. ${ }^{64}$ Bridging social capital represents connection between different types of actors, such as those in different groups, where as bonding social capital reflects homophily and connection between those with similar traits. Both these relationships can yield considerable power for those involved, with bridging social capital reflecting the strength of weak $\operatorname{ties}^{65}$ and bonding social capital reflecting a likelihood of leveraging cooperation. $^{66}$

Particularly in chaotic, dynamic or unstable environments, there is a considerable evolutionary incentive to gain advantage by closely monitoring adversaries that may exist ingroup. Sustaining network knowledge comes from sizing up other group members; so called social comparison is the process of judging third parties relative to oneself, which is used by humans to inform decision making, feelings and perceptions of ourselves and group members. Research in this area dates back to Festinger, ${ }^{67}$ and it has expanded to become an important field in its own right. ${ }^{68}$ It is dependent on a persistent frame of reference provided by ones own view of the world, where cues are used to understand the reputation and standing of ones counterparts, and may arise through numerous means including gossip. ${ }^{69}$ The dimensions through which social comparison is undertaken also gives insight into the nature of the group's norms, beliefs and values (Section 3.1).

The widespread disposition to undertake social comparison points to its important evolutionary role in sustaining human groups. ${ }^{70}$ It is phylogenetically ancient ${ }^{71}$ and embedded in human survival, with its suggested origins in evaluating competitors and assessing whether or not to commit resources to challenge a rival in the groups' hierarchy. Skills in social comparison also provide an advantage in understanding ones social position and the relationships to prioritise, ${ }^{71}$ which is of high importance when choosing to donate limited resources in support of another. Continued re-assessment of comparators and deliberation over observed social positioning in large groups requires significant cognitive resources relative to other species, ${ }^{72}$ consistent with the unusually large brain size in humans compared to other all other species ${ }^{73}$ and indicating our predisposition to this.

These cognitive skills are likely to be well-exercised by members of groups where instability is commonplace and violence is used in rivalry to control relationships and assert status. Equally in authoritarian regimes, challenges to power may typically result in responses to brutally reinforce control over rivals. Therefore the social and political context of power, and the agency held by individuals, informs the potential and way in which groups will evolve.

\subsection{Ingroup bias}

A consequence of evolutionary selection based on successful groups is a well-recognised disposition for humans to disproportionately preference the characteristics of their own group, known as ingroup bias. Such characteristics include a disposition that ones own group may hold the "correct" world view, while assuming the values of held by others do not have the same legitimacy or are somehow flawed. Confidence in superiority of the ingroup's capabilities is also reflected through this phenomenon. ${ }^{74,75}$ The disposition toward ingroup bias has emerged as an evolutionary consequence of reducing risk by preference for those with which stronger ties are held. 
A longstanding literature captures evidence of ingroup bias, and it is well-known for its potentially disruptive effects $^{76}$ while also being promoted through natural selection. ${ }^{15}$ ingroup bias functions as a form of social comparison at group level, with the comparison being made against the outgroup. This phenomenon of distinguishing between the ingroup and outgroup has been fundamental to developing what is arguably the most comprehensive psychological theory that explains intergroup conflict, ${ }^{24}$ which captures status rivalry between groups.

Tajfel et al's theory of intergroup conflict considers a continuum along which individuals interact: described at one extreme as interpersonal, where individual interactions are independent of group affiliations, verses intergroup, where interactions between individuals are fully determined by the group affiliations of the associated individuals. It is observed that the more extreme the conflict, the more likely individuals will be represented by their respective group identities. The theory identifies a psychological rationale that contribute to conflict.

The theory characterises inter-group conflict by considering the agency that individuals in a society have for movement between groups (i.e., the extent of social mobility verses barriers from stratification). This is deemed important because lack of individual agency will encourage individuals to consider themselves through the groups that they are associated with, rather than through their own individual identities. Intergroup conflict aligns with low social mobility, where greater uniformity of individuals through adoption of group rather than individual identities will be shown to members of their relevant outgroup.

Scenarios where Tajfel et al's theory identifies conflict emerging are consistent with the profound work of Gordon Allport, ${ }^{77}$ who proposed that prejudice between groups could be diminished by contact between members of those groups. This was deemed effective when interacting individuals have equal status, are cooperative, have common goals and are supported by underlying institutions. Considerable further development of Intergroup Contact Theory has since been accomplished, with Pettigrew ${ }^{78}$ identifying four change processes based on learning about the outgroup, changing behaviour to allow interaction, creating opportunity for human-to-human relationships and reconsideration of the ingroup.

Contributions characterising inter-group conflict and the role of contact can be used to interpret the extent of integration that a group possesses and to what extent this represents a threat. The extent to which individuals adopt the group identity, and the nature of the group's social norm and cooperative stance (Section 3.5) combine to provide assessments that over time may signal the extent of tensions emerging. The relationship between an individuals identity and their group identity has become an important emerging focus in the literature ${ }^{79}$ with identity fusion theory providing a useful framework. This focuses on the interplay between an individuals personal identity (who I am) and their social identity (the group to which I belong), assessing the permeability and interplay between these two factors. This impacts on the extent to which individuals categorize themselves and others through their group affiliation. Being able to characterise the nature and level of outgroup distortion that a group possesses, in terms of affective, behavioural and cognitive signals is therefore useful when assessing changes to a group's role in relation to others.

\subsection{Cooperation}

Cooperation is an inherently human disposition and is fundamental to sustaining coalitions, which represent alliances for mutual benefit to those involved and allow groups to function. Evolution has provided genetic wiring, in the form of the social brain (Section 2.1) that results in neurological effects from cooperation actions. ${ }^{80}$ How humans exhibit cooperative behaviour, with whom and in what form provides insight into the nature of groups to which they belong. Additionally, beyond defection and shirking, acts of conflict sit polar opposite to cooperation, involving resource removal or destruction rather than donating resources to others. Therefore understanding cooperation and conflict are very much related through this continuum of behaviour.

The potential cooperative behaviour of individuals in a group can also be thought of as finite resource, combining time and cognitive capability to maintain relationships with others. Therefore how actions are chosen, the extent of focus ingroup or outgroup and the positivity, negativity or extremism that is associated with behaviour strongly characterise the nature of a group. Whether actions are associated with individual identity or group identity may be challenging to assert, but it resonates with observations made in Tajfel's intergroup contact theor ${ }^{24}$ where extreme behaviour is more likely associated with use of a group identity by individuals. 
Importantly, cooperation brings together all the previous dimensions of groups as considered in this Section, and represents the basis around which human groups are sustained. Social norms (Section 3.1) relate to expectations concerning cooperation with others, extending to hostile and destructive acts, concerning both the ingroup and outgroup (Section 3.4), as well as the role of punishment when norms aren't followed. Status rivalry (Section 3.3 ) is dependent on the social capital available to individuals and is governed by the relationships through which significant cooperation may be levered. Individual standing (Section 3.2) indicates how people will be judged as a consequence of their actions towards others, which involves assessing their cooperative and hostile behaviour and to whom it is focussed (Section 3.4). Therefore characterising cooperative and uncooperative behaviour, and the associated motivations, are fundamental, providing the building blocks for assessment and modelling.

In the academic literature, identifying the natural conditions that allow cooperation to prosper has been a considerable research focus, spanning theoretical biology ${ }^{81}$ through to sociology ${ }^{47}$ and economics. ${ }^{82}$ However this has largely been accomplished in absence of groups or unstable geo-political scenarios but provides some useful fundamental principles. The most relevant forms of cooperation concerning groups are: direct reciprocity, ${ }^{83,84}$ which has some relation to social exchange theory in sociology ${ }^{85}$ where the receiver of a donation chooses whether or not to reciprocate; indirect reciprocity ${ }^{86}$ which is also related to generalized exchange theory ${ }^{87}$ where the reciprocation of a cooperative act arises from a wider population, and strong reciprocity, ${ }^{50}$ which involves punishment by group members for divergence from a group's particular cooperative social norm. These cooperative phenomena lend themselves to quantitative assessment and modelling, classically taking the form of game theory, which focuses on behaviour in the context of rational individual objectives, absence of any social reasoning. This does not render game theory an inappropriate tool, but motivates its adaptation to incorporate the social strategies that individuals may pursue as a consequence of their presence in particular groups. ${ }^{88}$

In some sense we can view cooperation as a fundamental way in which the nature of groups can be assessed and studied on a generative basis. Cooperation has the characteristic of an "atomic element" in the composition of a group because all behaviours can be judged in this dimension. This does not diminish the importance of effects and dynamics seen upon groups, such as communication, propaganda and influence propagation, as these forces may result in effects which shape or change the nature of a group's cooperative characteristics. A challenge however remains in detecting events, particularly those of a smaller scale, that represent indicators of behaviour that are difficult to observe through their latent nature. Although noisy and disconnected from physical behaviour, social media can provide additional input to the situational understanding of group behaviours, but requires significant knowledge fusion in the sense that integration needs to be achieved with human knowledge. However dimensions of mutability offer a potential framework to assess social media to comprehend the role and significance of communication combined with in-the-field observation. This is considered further in Section 4.

\section{EXAMPLES OF ANALYSIS USING THE PROPOSED DIMENSIONS OF MUTIBILITY}

In this section we summarise how the proposed five dimensions of mutibility (Section 3) can relate to the observed characteristics of groups. Firstly we observe that external forces that are known to affect groups can be interpreted in terms of the proposed five dimensions. The scope of each dimension in respect of changes to a group is summarized in Table 1: this presents the type of influence that each dimension may be associated with, in terms of role and scope. The table shows how different scales of resolution, from the individual actor through to the internal and external group levels, are concurrently important. Table 3 presents the effects of three commonly considered external forces that affect how groups function in scenarios related to instability. These concern access to resources, inequality and political failures. The consequences of these forces on groups are presented for each of the five dimensions of group mutability, manifesting themselves in slightly different ways and with different emphasis, depending on the scenario. These are hypothetical scenarios that demonstrate how group behaviours may be interpreted and provide insight. Using the proposed dimensions of mutability helps conceptual organization of the effects without supressing the associated complexity of the phenomenon.

\subsection{Situational Understanding and Social Media Data}

Alongside the often-chaotic organization on the ground, extremist groups and members are using social media to reinforce their position. Twitter in particular, because of its emphasis on short, easily-consumable, and real-time 
Table 1. Preliminary role and scope of dimensions of mutability in assessing groups.

\begin{tabular}{|l|l|l|}
\hline DIMENSION & $\begin{array}{l}\text { REFLECTION IN GROUP } \\
\text { CHARACTERISITICS }\end{array}$ & $\begin{array}{l}\text { PROVIDES } \\
\text { BOUNDARIES FOR: }\end{array}$ \\
\hline Social Norms & The general outlook of a group & $\begin{array}{l}\text { World view of values, beliefs, social } \\
\text { constraints and sanctions }\end{array}$ \\
\hline Cooperation & $\begin{array}{l}\text { Disposition in terms of aiding or } \\
\text { opposing others }\end{array}$ & $\begin{array}{l}\text { Acts of participation or expression } \\
\text { of conflict }\end{array}$ \\
\hline Ingroup Bias & $\begin{array}{l}\text { Categorisation of others and } \\
\text { homophilic attraction }\end{array}$ & $\begin{array}{l}\text { Shared identity and extent of similarity } \\
\text { and differentiation towards others }\end{array}$ \\
\hline Status Rivalry & $\begin{array}{l}\text { Ingroup hierarchy and influential } \\
\text { connection to others }\end{array}$ & $\begin{array}{l}\text { Social power from positioning, } \\
\text { inequality, resources and control }\end{array}$ \\
\hline Individual Standing & $\begin{array}{l}\text { The currency (e.g., capability, actions, } \\
\text { resources) that individuals possess to } \\
\text { gain social traction within their group }\end{array}$ & $\begin{array}{l}\text { Reputation and how members are } \\
\text { judged by peers to improve their } \\
\text { ingroup status }\end{array}$ \\
\hline
\end{tabular}

posts, has emerged as a key source of real-time 'breaking' information, often serving as a carrier of links to other channels, such as mainstream media news items. ${ }^{89}$ The real-time public dissemination of information (and misinformation) via social media is having significant impact on the practice of intelligence analysis in relation to the activities of online groups and movements ${ }^{90}$ and management of large-scale events. ${ }^{91}$ Consequently, the ability to rapidly process and exploit open source information in the context of understanding group behaviour dynamics has grown in significance in recent years.

Table 2. Interpreting social media signals in a group context.

\begin{tabular}{|l|l|}
\hline DIMENSION & POTENTIAL SIGNALS AND CONTENT FROM SOCIAL MEDIA \\
\hline Social Norms & $\begin{array}{l}\text { Reinforcement of beliefs, values and views. } \\
\text { Exposure of threats, sanctions and use of propaganda. }\end{array}$ \\
\hline Cooperation & $\begin{array}{l}\text { Promotion and reinforcement of participation within a group. Expressions } \\
\text { of opposition, threats and hatred to reinforce conflict and social norms. }\end{array}$ \\
\hline Ingroup Bias & $\begin{array}{l}\text { Targeted discrimination to entrench boundaries between groups. Expressions } \\
\text { of superiority of the ingroup and inferiority of the outgroup. }\end{array}$ \\
\hline Status Rivalry & $\begin{array}{l}\text { Expressions of commitment and following to particular individuals in the } \\
\text { group. Emphasis of differentiation between ingroup competitors. }\end{array}$ \\
\hline Individual Standing & $\begin{array}{l}\text { Content representing the currency that groups recognise in terms of } \\
\text { reputation, status and respect from inside the group. }\end{array}$ \\
\hline
\end{tabular}

There are now diverse tools for assessing social media content, which generally provide an observatory function. While such technology plays a key element in open source intelligence exploitation, especially in the area of social sensing, ${ }^{92}$ a tendency to 'oversell' its capabilities has been observed ${ }^{93,94}$ leading to a view that 'glass box' rather than 'black box' approaches are required in order to gain robust situational understanding from open source media. ${ }^{95,96}$ For example, an analysis of the failure of Google's 'Flu Trends' algorithm in early 2013 pointed to a lack of transparency in both algorithms and social media platforms as being key factors in undermining reliability and replicability of analysis results over time. ${ }^{94}$ Addressing this issue in terms of supporting situational understanding in relation to group behaviours and activity requires robust models of group dynamics and in particular mutability. 


\begin{tabular}{|c|c|c|c|}
\hline 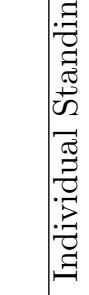 & 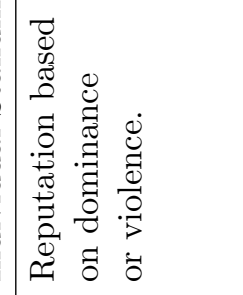 & 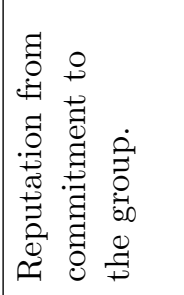 & 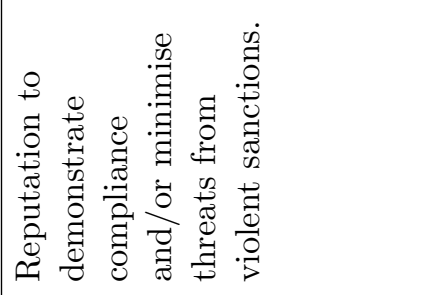 \\
\hline 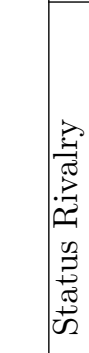 & 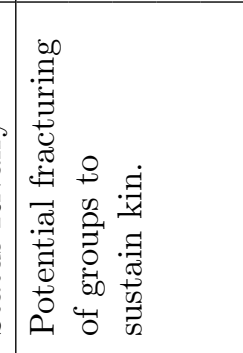 & 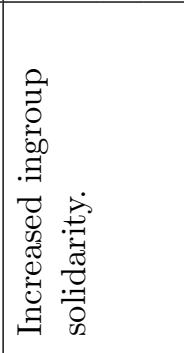 & 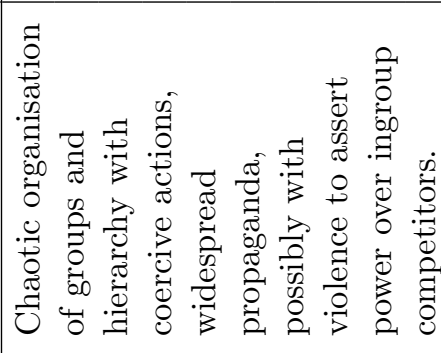 \\
\hline 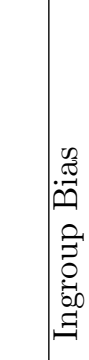 & 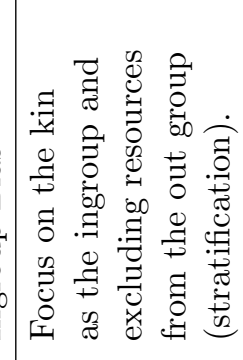 & 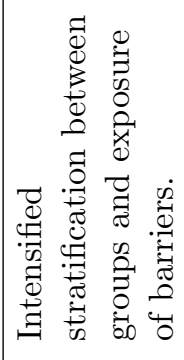 & 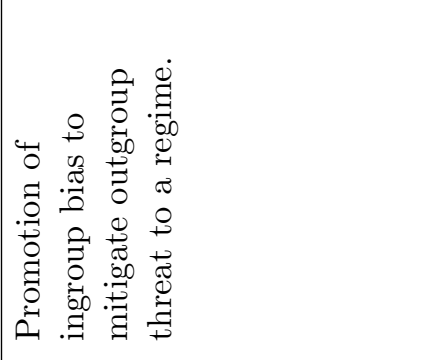 \\
\hline 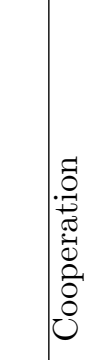 & 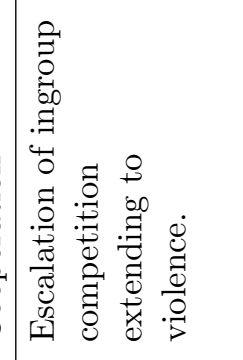 & 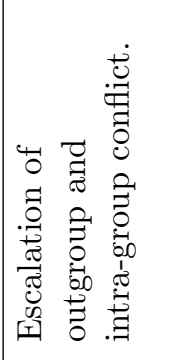 & 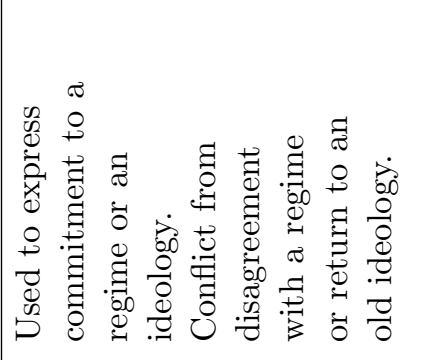 \\
\hline 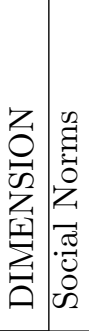 & 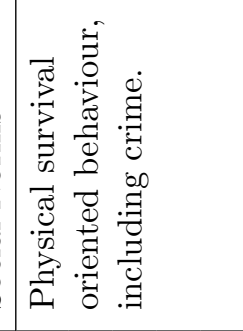 & 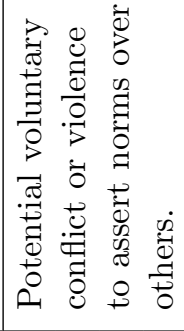 & 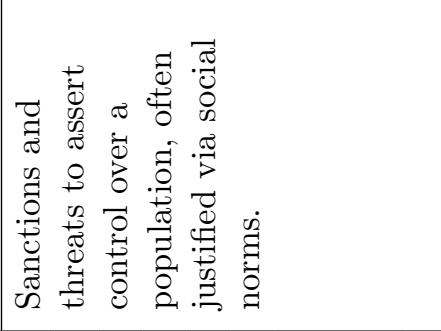 \\
\hline 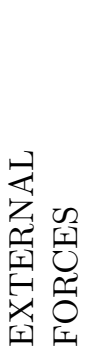 & 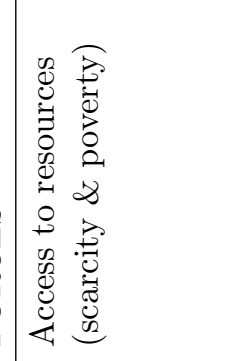 & 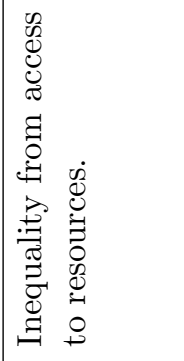 & 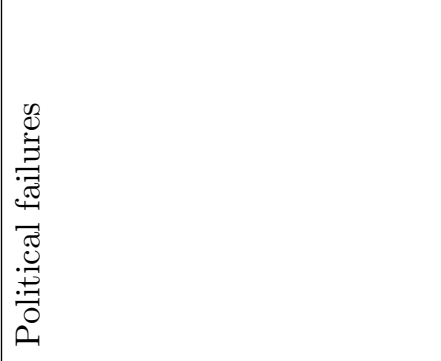 \\
\hline
\end{tabular}


A feature of this media is the inherent noise associated with typically short messaging and disconnection from the physical world. As a result interpreting the meaning of content and its relevance to groups is particularly challenging, leading to generally broad-brush approaches that may involve aspects such as sentiment analysis to glean overall characteristics around aggregated messaging, that might or might not correlate with activity on the ground. More generally, interpreting social media content in the context of group behaviour is a problematic issue. In Table 2, we show how the proposed dimensions of mutability can be used to help interpret and analyse online group behaviour. This allows the content involved in an individual social media post to be interpreted in terms of its contribution to group dynamics. Effectively this translates semantic analysis of social media to its role in a group, existing approaches for which are not well documented.

\section{CONCLUSIONS}

In this paper we have focused on developing and understanding characteristics of groups that can be used to assess changes in groups. Our motivation for considering groups as an important entity goes back to the anthropological literature, where groups have been influential in human evolution and survival. From the contemporary literature, diverse approaches have been made at modelling groups, each with different points of focus. To assist in understanding human groups, five dimensions have been proposed that allow complex dynamics to be related and understood without suppressing the complexity that is inherent in human groups. The organisation of group characteristics in this manner is useful because it provides a framework for the future development of generative models of group behaviour, as well as supporting the analysis and interpretation of changes to group behaviour.

\section{ACKNOWLEDGMENTS}

This research was sponsored by the U.S. Army Research Laboratory and the U.K. Ministry of Defence under Agreement Number W911NF-16-3-0001. The views and conclusions contained in this document are those of the authors and should not be interpreted as representing the official policies, either expressed or implied, of the U.S. Army Research Laboratory, the U.S. Government, the U.K. Ministry of Defence or the U.K. Government. The U.S. and U.K. Governments are authorized to reproduce and distribute reprints for Government purposes notwithstanding any copy-right notation hereon. Content includes material subject to Crown copyright (C)(2017), Dstl. This material is licensed under the terms of the Open Government Licence except where otherwise stated. To view this licence, visit http://www.nationalarchives.gov.uk/doc/open-governmentlicence/version/3 or write to the Information Policy Team, The National Archives, Kew, London TW9 4DU, or email: psi@nationalarchives.gsi.gov.uk. Dstl/CP101011

\section{REFERENCES}

1. L. F. Richardson, Arms and insecurity: A mathematical study of the causes and origins of war, Boxwood Press, 1960

2. P. K. Davis and K. Cragin, Social science for counterterrorism: Putting the pieces together, Rand Corporation, 2009.

3. A. Gat, War in human civilization, Oxford University Press, 2008.

4. G. Tononi, "An information integration theory of consciousness," BMC neuroscience 5(1), p. 1, 2004.

5. H. Tajfel, "Social identity and intergroup behaviour," Social Science Information/sur les sciences sociales , 1974.

6. P. Salovey and J. D. Mayer, "Emotional intelligence," Imagination, cognition and personality 9(3), pp. 185211,1990

7. B. Latané, "The psychology of social impact," American psychologist 36(4), p. 343, 1981.

8. A. Nowak, J. Szamrej, and B. Latané, "From private attitude to public opinion: A dynamic theory of social impact," Psychological Review 97(3), p. 362, 1990.

9. B. Latané, "Dynamic social impact: The creation of culture by communication," Journal of Communication 46(4), pp. 13-25, 1996.

10. R. Axtell, "Why agents?: on the varied motivations for agent computing in the social sciences," 2000. 
11. J. M. Epstein, "Agent-based computational models and generative social science," Complexity 4(5), pp. 4160, 1999.

12. M. W. Macy and R. Willer, "From factors to actors: Computational sociology and agent-based modeling," Annual review of sociology, pp. 143-166, 2002.

13. R. Axelrod, "An evolutionary approach to norms," American political science review 80(04), pp. 1095-1111, 1986.

14. R. Axelrod, "The dissemination of culture a model with local convergence and global polarization," Journal of conflict resolution 41(2), pp. 203-226, 1997.

15. R. A. Hammond and R. Axelrod, "The evolution of ethnocentrism," Journal of Conflict Resolution 50(6), pp. 926-936, 2006.

16. M. McPherson, "An ecology of affiliation," American Sociological Review , pp. 519-532, 1983.

17. N. Choucri, D. Goldsmith, S. Madnick, D. Mistree, J. B. Morrison, and M. Siegel, "Using system dynamics to model and better understand state stability," 2007.

18. M. L. Bernard, G. A. Backus, A. B. Bier, and S. F. Branch, "Behavioral influence assessment (bia): A multi-scale system to assess dynamic behaviors within groups and societies across time," Advances in CrossCultural Decision Making 5, p. 161, 2014.

19. A. B. Bier and M. L. Bernard, "Validating a hybrid cognitive-system dynamics model of team interaction," Advances in Cross-Cultural Decision Making 5, p. 209, 2014.

20. J. M. Epstein, Generative social science: Studies in agent-based computational modeling, Princeton University Press, 2006.

21. J. Landeta, "Current validity of the delphi method in social sciences," Technological forecasting and social change 73(5), pp. 467-482, 2006.

22. J. Tooby, "Coalitional instincts." https://www.edge.org/response-detail/27168. Accessed: 2017-06-01.

23. H. Tajfel, "Social psychology of intergroup relations," Annual review of psychology 33(1), pp. 1-39, 1982.

24. H. Tajfel and J. C. Turner, "An integrative theory of intergroup conflict," The social psychology of intergroup relations 33(47), p. 74, 1979.

25. R. I. M. Dunbar, Grooming, gossip, and the evolution of language, Harvard University Press, 1998.

26. E. Fehr, "Human behaviour: don't lose your reputation," Nature 432(7016), pp. 449-450, 2004.

27. R. Boyd and P. J. Richerson, "Culture and the evolution of human cooperation," Philosophical Transactions of the Royal Society of London B: Biological Sciences 364(1533), pp. 3281-3288, 2009.

28. F. W. Marlowe, "Hunter-gatherers and human evolution," Evolutionary Anthropology: Issues, News, and Reviews 14(2), pp. 54-67, 2005.

29. R. Byrne and A. Whiten, "Machiavellian intelligence: social expertise and the evolution of intellect in monkeys, apes, and humans (oxford science publications)," 1989.

30. J. P. Carpenter, "Punishing free-riders: How group size affects mutual monitoring and the provision of public goods," Games and Economic Behavior 60(1), pp. 31-51, 2007.

31. R. I. M. Dunbar, A. H. Korstjens, and J. Lehmann, "Time as an ecological constraint," Biological Reviews 84(3), pp. 413-429, 2009.

32. R. I. M. Dunbar, "The social role of touch in humans and primates: behavioural function and neurobiological mechanisms," Neuroscience \&6 Biobehavioral Reviews 34(2), pp. 260-268, 2010.

33. M. McPherson, L. Smith-Lovin, and J. M. Cook, "Birds of a feather: Homophily in social networks," Annual review of sociology, pp. 415-444, 2001.

34. E. Anderson, "Beyond homo economicus: New developments in theories of social norms," Philosophy E6 Public Affairs 29(2), pp. 170-200, 2000.

35. R. I. M. Dunbar and S. Shultz, "Evolution in the social brain," Science 317(5843), pp. 1344-1347, 2007.

36. R. I. M. Dunbar and S. Shultz, "Bondedness and sociality," Behaviour 147(7), pp. 775-803, 2010.

37. R. A. Hill and R. I. M. Dunbar, "Social network size in humans," Human nature 14(1), pp. 53-72, 2003.

38. H. Kudo and R. I. M. Dunbar, "Neocortex size and social network size in primates," Animal Behaviour 62(4), pp. 711-722, 2001.

39. R. I. M. Dunbar, "The social brain hypothesis," Evolutionary Anthropology , pp. 178-190, 1998. 
40. R. I. M. Dunbar, "Coevolution of neocortical size, group size and language in humans," Behavioral and brain sciences 16(04), pp. 681-694, 1993.

41. A. Whiten, Natural theories of mind: Evolution, development and simulation of everyday mindreading, Basil Blackwell Oxford, 1991.

42. B. F. Malle and J. Knobe, "The folk concept of intentionality," Journal of Experimental Social Psychology 33(2), pp. 101-121, 1997.

43. B. F. Malle, L. J. Moses, and D. A. Baldwin, Intentions and intentionality: Foundations of social cognition, MIT press, 2001.

44. S. T. Fiske, A. J. Cuddy, and P. Glick, "Universal dimensions of social cognition: Warmth and competence," Trends in cognitive sciences 11(2), pp. 77-83, 2007.

45. L. Schilbach, S. B. Eickhoff, A. Rotarska-Jagiela, G. R. Fink, and K. Vogeley, "Minds at rest? social cognition as the default mode of cognizing and its putative relationship to the default system of the brain," Consciousness and cognition 17(2), pp. 457-467, 2008.

46. G. C. Homans, The human group, vol. 7, Routledge, 2013.

47. G. C. Homans, "Social behavior as exchange," American journal of sociology , pp. 597-606, 1958.

48. G. Therborn, "Back to norms! on the scope and dynamics of norms and normative action," Current Sociology 50(6), pp. 863-880, 2002.

49. R. Boyd and P. J. Richerson, "The evolution of norms: An anthropological view," Journal of Institutional and Theoretical Economics (JITE)/Zeitschrift für die gesamte Staatswissenschaft, pp. 72-87, 1994.

50. E. Fehr, U. Fischbacher, and S. Gächter, "Strong reciprocity, human cooperation, and the enforcement of social norms," Human nature 13(1), pp. 1-25, 2002.

51. E. Fehr and U. Fischbacher, "Social norms and human cooperation," Trends in cognitive sciences 8(4), pp. 185-190, 2004.

52. R. Boyd and P. J. Richerson, "Group beneficial norms can spread rapidly in a structured population," Journal of theoretical biology 215(3), pp. 287-296, 2002.

53. M. A. Hogg, "Social identity theory," Contemporary social psychological theories 13, pp. 111-1369, 2006.

54. J. Horgan, "From profiles to pathways and roots to routes: Perspectives from psychology on radicalization into terrorism," The ANNALS of the American Academy of Political and Social Science 618(1), pp. 80-94, 2008.

55. R. A. Hudson and M. Majeska, "The sociology and psychology of terrorism: Who becomes a terrorist and why?," Library of Congress Washington, DC, 1999.

56. K. Izuma, "The social neuroscience of reputation," Neuroscience research 72(4), pp. 283-288, 2012.

57. M. Jensen, H. Kim, and B. Kim, "Meeting expectations: A role-theoretic perspective on reputation," The Oxford handbook of corporate reputation, pp. 140-159, 2012.

58. R. P. Beatty and J. R. Ritter, "Investment banking, reputation, and the underpricing of initial public offerings," Journal of financial economics 15(1), pp. 213-232, 1986.

59. D. Engelmann and U. Fischbacher, "Indirect reciprocity and strategic reputation building in an experimental helping game," Games and Economic Behavior 67(2), pp. 399-407, 2009.

60. M. Milinski, "Reputation, a universal currency for human social interactions," Phil. Trans. R. Soc. $B$ 371(1687), p. 20150100, 2016.

61. N. Lin, "Building a network theory of social capital," Connections 22(1), pp. 28-51, 1999.

62. R. S. Burt, Brokerage and closure: An introduction to social capital, Oxford university press, 2005.

63. P. Bourdieu, "The forms of capital.(1986)," Cultural theory: An anthology, pp. 81-93, 2011.

64. R. A. Baron, Social capital, Wiley Online Library, 2000.

65. M. Granovetter, "The strength of weak ties: A network theory revisited," Sociological theory 1(1), pp. 201$233,1983$.

66. J. H. Fowler and N. A. Christakis, "Cooperative behavior cascades in human social networks," Proceedings of the National Academy of Sciences 107(12), pp. 5334-5338, 2010.

67. L. Festinger, "A theory of social comparison processes," Human relations 7(2), pp. 117-140, 1954. 
68. A. P. Buunk and F. X. Gibbons, "Social comparison: The end of a theory and the emergence of a field," Organizational Behavior and Human Decision Processes 102(1), pp. 3-21, 2007.

69. S. R. Wert and P. Salovey, "A social comparison account of gossip," Review of General Psychology 8(2), p. $122,2004$.

70. R. M. Whitaker, G. B. Colombo, S. M. Allen, and R. I. M. Dunbar, "A dominant social comparison heuristic unites alternative mechanisms for the evolution of indirect reciprocity," Scientific Reports $\mathbf{6}, 2016$.

71. P. Gilbert, J. Price, and S. Allan, "Social comparison, social attractiveness and evolution: How might they be related?," New Ideas in Psychology 13(2), pp. 149-165, 1995.

72. R. D. Alexander, The biology of moral systems, Transaction Publishers, 1987.

73. R. A. Barton and R. I. M. Dunbar, "Evolution of the social brain," Machiavellian intelligence II: Extensions and evaluations 2, p. 240, 1997.

74. H. Tajfel, "Experiments in intergroup discrimination," Scientific American 223(5), pp. 96-102, 1970.

75. H. Tajfel, M. G. Billig, R. P. Bundy, and C. Flament, "Social categorization and intergroup behaviour," European journal of social psychology 1(2), pp. 149-178, 1971.

76. S. T. Fiske, "What we know now about bias and intergroup conflict, the problem of the century," Current Directions in Psychological Science 11(4), pp. 123-128, 2002.

77. G. W. Allport, The nature of prejudice, Cambridge/Reading, MA: Addison-Wesley, 1954.

78. T. F. Pettigrew, "Intergroup contact theory," Annual review of psychology 49(1), pp. 65-85, 1998.

79. W. B. Swann Jr, J. Jetten, A. Gómez, H. Whitehouse, and B. Bastian, "When group membership gets personal: a theory of identity fusion.," Psychological review 119(3), p. 441, 2012.

80. A. G. Sanfey, "Social decision-making: insights from game theory and neuroscience," Science 318(5850), pp. 598-602, 2007.

81. M. A. Nowak, "Five rules for the evolution of cooperation," science 314(5805), pp. 1560-1563, 2006.

82. H. Gintis, Moral sentiments and material interests: The foundations of cooperation in economic life, vol. 6 , MIT press, 2005.

83. A. Rapoport and A. M. Chammah, Prisoner's dilemma: A study in conflict and cooperation, vol. 165, University of Michigan press, 1965.

84. R. M. Axelrod, The evolution of cooperation, Basic books, 2006.

85. K. S. Cook, C. Cheshire, E. R. Rice, and S. Nakagawa, "Social exchange theory," in Handbook of social psychology, pp. 61-88, Springer, 2013.

86. K. Panchanathan and R. Boyd, "Indirect reciprocity can stabilize cooperation without the second-order free rider problem," Nature 432(7016), pp. 499-502, 2004.

87. N. Takahashi, "The emergence of generalized exchange," American Journal of Sociology , pp. 1105-1134, 2000 .

88. D. Rohner, "Reputation, group structure and social tensions," Journal of Development Economics 96(2), pp. 188-199, 2011.

89. F. Abel, C. Hauff, G.-J. Houben, R. Stronkman, and K. Tao, "Semantics + filtering + search = Twitcident. exploring information in social web streams," in Proceedings of the 23rd ACM Conference on Hypertext and Social Media, pp. 285-294, 2012.

90. M. Innes, C. Roberts, and D. Rogers, "Critical timing," Police Professional January, pp. 17-18, 2014.

91. C. Roberts, M. Innes, A. Preece, and I. Spasić, "Soft facts and spontaneous community mobilisation: the role of rumour after major crime events," in Data for Good: How big and open data can be used for the common good, P. Baeck, ed., pp. 37-43, Nesta, 2015.

92. D. Wang, T. Abdelzaher, and L. Kaplan, Social Sensing: Building Reliable Systems on Unreliable Data, Morgan Kaufmann, 2015.

93. D. Gayo-Avello, "A meta-analysis of state-of-the-art electoral prediction from twitter data," Social Science Computer Review 31(6), pp. 649-679, 2013.

94. D. Lazer, R. K. G. King, and A. Vespignani, "The parable of Google Flu: Traps in big data analysis," Science 343, pp. 1203-1205, 2014.

95. M. Innes, C. Roberts, A. Preece, and D. Rogers, "Of instruments and data: Social media uses, abuses and analysis," in Handbook of Online Research Methods, N. Fielding, ed., Sage, 2 ed.

96. F. Pasquale, The Black Box Society, Harvard University Press, 2015. 